PAPER

\title{
Repetitive training of compensatory steps: a therapeutic approach for postural instability in Parkinson's disease
}

\author{
M Jöbges, G Heuschkel, C Pretzel, C Illhardt, C Renner, H Hummelsheim
}

J Neurol Neurosurg Psychiatry 2004;75:1682-1687. doi: 10.1136/jnnp.2003.016550

See end of article for authors' affiliations

.....................

Correspondence to: Dr M Jöbges, NRZ Leipzig, Muldentalweg 1, 04828 Bennewitz, Germany; joebges@sachsenklinik.de

Received 9 April 2003 Accepted in revised form 22 March 2004
Background: Postural instability (PI) is a common and serious problem in Parkinson's disease (PD). Dopaminergic medication is of negligible use and a positive effect of deep brain stimulation on this issue has not been reported.

Objective: To develop a method of repetitive training of compensatory steps to enhance protective postural responses by using training strategies based on recent neurophysiological research.

Methods: Fourteen patients with PD took part in a multiple baseline design study and were trained for 14 days in an ambulant setting consisting of two daily sessions.

Results: After training, the length of compensatory steps increased and the step initiation shortened. In a gait analysis, the cadence and the step length increased, gait velocity improved, and the period of double support shortened. The "mobility" subscore of a quality of life questionnaire (PDQ-39) also improved. All these changes were significant $(p<0.05)$. These effects were stable for two months without additional training.

Conclusion: The repetitive training of compensatory steps is an effective approach in the treatment of PI and should be applied if $\mathrm{PI}$ is evident clinically or in a patient's history.
$\mathrm{P}$ ostural instability is a common and serious problem in Parkinson's disease (PD). Postural reactions of up to $96 \%$ of all parkinsonian patients diminish during the course of the disease. ${ }^{1}$ Koller and colleagues ${ }^{2}$ reported that 38 of 100 patients with PD fall-13\% of them more than once a week$13 \%$ experience fractures, $18 \%$ hospitalisation, and 3\% are confined to a wheelchair. In addition, social isolation occurs because of the fear of walking. ${ }^{12}$ Unfortunately, the effect of dopaminergic medication on postural instability is negligible, ${ }^{3}$ and can even worsen computerised dynamic posturography tests. ${ }^{4}$ The innovative deep brain stimulation targets tremor, off motor symptoms, and dyskinesias, ${ }^{5}$ whereas an improvement of postural instability is not reported. Therefore, lesions outside the dopaminergic system are thought to contribute to the pathophysiology of postural instability in PD. ${ }^{3}$ Furthermore, postural instability is not restricted to the late stages of the disease, and it can even be the first presenting symptom. ${ }^{16}$

Postural responses can be classified as corrective or protective. $^{7}$ Corrective responses are characterised by a constant base of support; equilibrium is achieved by the activation of leg, trunk, and neck muscles. In protective responses, the base of support changes-for example, with the execution of a compensatory step. Both kinds of responses are reduced in PD. In light of the unsatisfactory influence of dopaminergic drugs, the development of nonpharmacological therapeutic approaches is warranted to compensate for postural instabilities and to minimise their sequelae.

Recent neurophysiological research in stroke patients suggests that repetitive motor activity forms an important prerequisite of motor learning. ${ }^{8}$ Our investigation evaluates a repetitive postural training method that teaches patients to perform compensatory steps and relates it to balance, gait, and quality of life.

\section{METHODS}

\section{Subjects}

Our study comprised 14 outpatients with PD (eight women and six men; mean age, 60.7, age range, 41-75 years), diagnosed according to the UK Parkinson's Disease Society Brain Research Centre criteria9 (table 1). The patients were recruited from a PD self help association. Only patients with PD suffering from postural instability were asked to participate. The investigators were not the treating physicians or physiotherapists, and neither were they involved in the medical treatment or the physical treatment. The whole study course was explained to the patients in detail, there was an opportunity to ask questions, and participation was completely voluntary. Rejection of participation was without consequences for the patients.

The diagnosis of idiopathic PD was confirmed by a careful neurological examination. To obtain the Unified Parkinson's Disease Rating Scale (UPDRS) scores the patients fasted from midnight and took their last anti-Parkinson medicine not less than 12 hours before the test, which was performed around $9.00 \mathrm{am}$. First, the UPDRS "defined off" state data were taken, and then the patients received their usual medication. Approximately one hour later, when they reported their best "on" state, the UPDRS "defined on" score was obtained. The course of the disease ranged from two to 21 years and L-dopa was administered in doses from 0 to $1025 \mathrm{mg} /$ day (table $\mathrm{l}$ ). The total UPDRS ${ }^{10}$ score was between 16 and 91 in "defined off" and 16 and 79 in "defined on", with UPDRS III scores between 6 and 33 in "defined off" and 0.5 and 21 in "defined on" (according to the core assessment program for surgical interventional therapies in Parkinson's disease criteria ${ }^{11}$ ). The Hoehn and Yahr stages $^{10}$ ranged between 2.5 and 4 . The Schwab and England ${ }^{10}$ score was between 30\% and $90 \%$ in "defined off", and between $60 \%$ and $90 \%$ in "defined on". See table 1 for the exact individual classifications of each patient.

\section{Training}

We used a multiple baseline study design across individuals. ${ }^{12}$ The duration of the baseline phase was randomly assigned to

Abbreviations: LOS, limits of stability test; PD, Parkinson's disease; SOT, sensory organisation test; UPDRS, Unified Parkinson's Disease Rating Scale 
Table 1 Patient characteristics

\begin{tabular}{|c|c|c|c|c|c|c|c|c|c|c|}
\hline \multirow[b]{2}{*}{ Patient } & \multirow[b]{2}{*}{ Sex } & \multirow{2}{*}{$\begin{array}{l}\text { Age } \\
\text { (years) }\end{array}$} & \multirow{2}{*}{$\begin{array}{l}\text { Disease duration } \\
\text { (years) }\end{array}$} & \multicolumn{2}{|c|}{ UPDRS } & \multirow[b]{2}{*}{ H\&Y } & \multicolumn{2}{|l|}{ S\&E } & \multirow{2}{*}{$\begin{array}{l}\text { L-dopa dose } \\
\text { (mg/day) }\end{array}$} & \multirow[b]{2}{*}{ Dopamin agonist } \\
\hline & & & & On & Off & & On & Off & & \\
\hline 1 & $\mathrm{~F}$ & 74 & 7 & 51 & 51 & 3 & $80 \%$ & 80 & 250 & Dihydroergocriptine \\
\hline 2 & $\mathrm{~F}$ & 73 & 15 & 79 & 86 & 4 & $70 \%$ & 50 & 600 & Ropinirole \\
\hline 3 & $\mathrm{~F}$ & 60 & 3 & 37 & 42 & 2.5 & $90 \%$ & 80 & 250 & Cabergoline \\
\hline 4 & $\mathrm{~F}$ & 70 & 6 & 77 & 91 & 4 & $70 \%$ & 50 & 350 & Pergolide \\
\hline 5 & $\mathrm{~F}$ & 78 & 12 & 24 & 27 & 2.5 & $80 \%$ & 70 & 400 & Pergolide \\
\hline 6 & $\mathrm{~F}$ & 65 & 21 & 20 & 38 & 3 & $90 \%$ & 60 & 1025 & Pramipexole \\
\hline 7 & $M$ & 65 & 7 & 69 & 81 & 4 & $60 \%$ & 30 & 550 & Pramipexole \\
\hline 8 & $\mathrm{~F}$ & 78 & 3 & 50 & 50 & 2.5 & $80 \%$ & 80 & 0 & Pramipexole \\
\hline 9 & $\mathrm{~F}$ & 81 & 2 & 16 & 16 & 2.5 & $90 \%$ & 90 & 0 & Dihydroergocriptine \\
\hline 10 & $M$ & 78 & 6 & 60 & 67 & 3 & $70 \%$ & 50 & 600 & Pramipexole \\
\hline 11 & $\mathrm{~F}$ & 77 & 8 & 37 & 48 & 3 & $80 \%$ & 60 & 350 & Dihydroergocriptine \\
\hline $12^{*}$ & $M$ & 80 & 9 & 37 & 37 & 2.5 & $80 \%$ & 80 & 250 & Lisuride \\
\hline $13^{*}$ & $M$ & 70 & 5 & 44 & 44 & 2.5 & $80 \%$ & 80 & 500 & Cabergoline \\
\hline $14^{*}$ & $\mathrm{~F}$ & 72 & 3 & 34 & 40 & 2.5 & $80 \%$ & 80 & 400 & \\
\hline
\end{tabular}

*Patients dropped out after training.

F, female; H\&Y, Hoehn and Yahr stage; M, male; S\&E, Schwab and England score; UPDRS, Unified Parkinson's Disease Rating Scale, version 3.0.

each patient and lasted between one and three weeks. The baseline phase was followed by a two week training phase, with repetitive postural training for 20 minutes twice daily. Weekends were excluded, so the training was done for 10 days. A single physiotherapist was in charge of training all the patients during their training phase. This training consisted of repetitive pulls to the patient's back and pushes to her or his right and left side applied by the physiotherapist. The strength of the pulls and pushes was adapted to the degree of the patient's individual postural instability. In the case of satisfactory compensatory steps, a positive feedback was given, and the intensity of the pulls and pushes was continuously increased. Insufficient compensatory steps were corrected and, if necessary, the intensity of pulls and pushes was reduced. In the later stages of the training, the direction of the pushes was randomised. Within these 20 minute training sessions, approximately 180 to 230 pulls and pushes were applied. The training was directed on holding stability after the pushes by large compensatory steps.

\section{Evaluation}

Analysis of compensatory steps, posture, and gait was carried out before and after the baseline phase, after the end of the

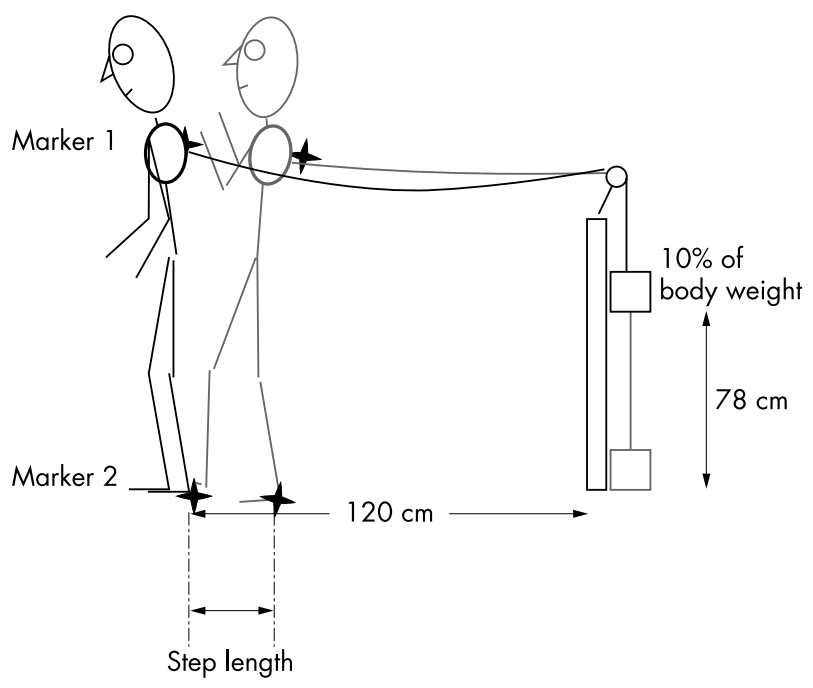

Figure 1 Experimental setup and marker location. Marker 1 is attached at the rope near the harness, marker 2 at the foot. training phase, and two weeks and two months later. Furthermore, the Parkinson's disease questionnaire (PDQ39) was used at the same intervals. Patients were always assessed at the same time of the day and at the same interval after the intake of their last medication. Medication was kept stable throughout the study.

Length and initiation of the compensatory steps were recorded using an ultrasound device (CMS 50; Zebris, Isny, Germany) that continuously calculates the three dimensional spatial position of two extremely small markers (fig 1) attached to the foot (marker 2), via flexible cables, and to a harness (marker 1, both markers sending out ultrasound signals with a frequency of $22 \mathrm{~Hz}$ each) connected by a rope. The other end of the rope was attached to a weight, which was $10 \%$ of the body weight of the individual patient. This weight was dropped from a $78 \mathrm{~cm}$ height to imitate a push leading to postural instability. The experimental set up is illustrated in fig 1. Step initiation was defined as the time of the first movement of marker 1 subtracted from the time of the first movement of marker 2. Step length was indicated by the change of position of marker 2. To measure the compensatory step towards the rear, the rope was fixed at the back; for measurement of compensatory steps to the right and left, the rope was fixed at the corresponding side of the harness. Each direction was tested five times. Patients were instructed to make large compensatory steps.

Gait analysis was carried out by means of the Zebris Win Gait system (Win Gait 2.14; Zebris). The patients had to walk three times over a distance of $5 \mathrm{~m}$. The measurement started after a walking distance of $1.5 \mathrm{~m}$, so the first steps were excluded.

The measuring process is based on a technical ultrasound pulse time measurement. In this method, small ultrasound markers were attached to the thigh, knee, ankle, and feet, on both sides of the body. Each of these eight ultrasound markers sends ultrasound signals with a frequency of $20 \mathrm{~Hz}$; the positional accuracy is $2 \mathrm{~mm}$. Signals from the left and the right side of the body were measured simultaneously. The time segments to be analysed were selected interactively. Step length, cadence, and double support were measured by means of the Zebris Win Gait system. The gait trajectories were displayed; gait cycles were automatically proposed and carefully checked and changed if necessary by an experienced investigator. After that, step length, cadence, and double support were automatically calculated (WinGait 2.14 for Windows; Zebris).

Posturographical testing was performed using a commercial balance platform (Balance Master; NeuroCom 


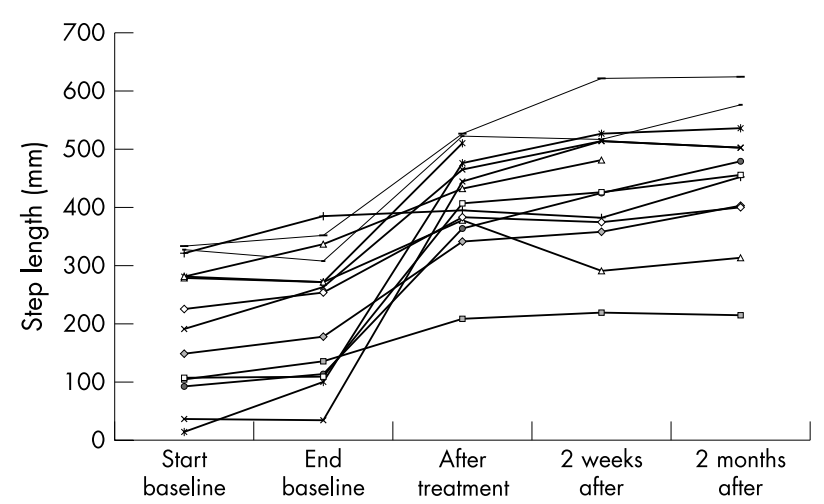

Figure 2 Length of compensation step in $\mathrm{mm}$; each line represents one patient. The length of the compensation step increased significantly after treatment.

International, Clackamas, Oregon, USA) to record vertical and horizontal shear forces and to allow calculations of the centre of gravity over time. Several tests were carried out covering static and dynamic components of postural stability. First, the "sensory organisation test" (SOT) measures the sway of the patient's centre of gravity under the following conditions: eyes open, firm platform; eyes closed, firm platform; eyes open, foam platform; eyes closed, foam platform. Second, the "limits of stability test" (LOS) measures the path of the sway, time, and distance travelled by the patient's centre of gravity from the starting place to eight different points (forward, backward, left, right, forward left, forward right, backward left, backward right) set at 50\% of the theoretical limit of stability of a control group (Balance Master Manual; NeuroCom International). The patients were given visual feedback on a computer screen reflecting the actual position of their centre of gravity. For both SOT and LOS, three trials of each condition were taken for later analysis. All tests were explained in detail to the patients and they were given several trials to familiarise themselves with the task.

The PDQ-39, a quality of life questionnaire developed for patients with PD, was carried out at the same time as the motor tests. The total score and the "mobility" subscore were taken for further analysis. ${ }^{13}$

Following the criteria of a multiple baseline study across individuals, ${ }^{12}$ the above mentioned parameters were measured at the beginning and at the end of the baseline phase, at the end of the subsequent period of repetitive postural training, and at two weeks and two months after the end of the training. This is a case-control study design where each patient's baseline data are compared to his/her treatment data. If the measured parameters change in a temporal relation to the intervention-the postural training-this effect can be attributed to the intervention rather than to extraneous events. To compare the data of one phase with the data of the preceding phase, a check of normal distribution was carried out. The $t$ test was used to analyse parametric data and the Wilcoxon signed rank test was used for nonparametric data to test the hypothesis that their distribution was the same. The level of significance was set at $p=0.05$. Because three patients dropped out during the follow up, comparisons of data between the start and the end of baseline and directly after treatment were carried out on 14 patients, whereas comparisons of data directly after treatment, two weeks, and two months after treatment used data from 11 patients.

Measurement values are reported as mean (SD).

\section{RESULTS}

The repetitive postural training was carried out in two daily sessions, one in the morning and one in the afternoon, with sufficient time in between to allow the participants to recover. The training was well tolerated by all patients. Only a small number of patients reported muscle stiffness in the proximal muscles of the leg, probably as a result of the unusual demand on these muscle groups. The muscular stiffness disappeared in all cases during the course of the training phase, and no patient reported it as disturbing. Three patients dropped out during the follow up period, two because of severe medical problems not related to the training, and one because of a lack of motivation. Medication was kept stable throughout our study.

\section{Compensatory steps}

During the baseline phase, the mean length of the first compensatory step after the pushing showed a small, albeit significant, increase from $195 \mathrm{~mm}$ to $222 \mathrm{~mm}$ (SD, 112 and $110 \mathrm{~mm}$, respectively; $\mathrm{p}=0.009 ; t$ test). At the end of the two week training phase, however, a prominent and significant increase of the compensatory step length was seen (mean, $418 \mathrm{~mm}$; SD, 85; $\mathrm{p}<0.001$; $t$ test) in comparison with the end of the baseline phase. In a follow up measurement two months after the end of the training, a step length of $450 \mathrm{~mm}$ (SD, 117) was registered, significantly longer than that seen directly after treatment $(\mathrm{p}=0.009$; $t$ test) (fig 2).

The delay until the initiation of the compensatory step remained stable during the baseline phase (mean, $651 \mathrm{~ms}$ at the start and end of the baseline phase; SD, 251 and 180, respectively), but shortened significantly (mean, $456 \mathrm{~ms}$; SD, 90; $\mathrm{p}=0.001 ; t$ test) during the training phase. Two months later, step initiation was further significantly shortened (mean, 331 ms; SD, 42; p $<0.001 ; t$ test) (fig 3).

\section{Gait analysis}

Gait analysis showed no significant changes of step length during walking before (mean, $0.77 \mathrm{~m}$; SD, 0.29) and after the baseline (mean, $0.80 \mathrm{~m}$; SD, 0.22), whereas a significant increase in step length occurred during the training phase (mean, $0.89 \mathrm{~m}$; SD, 0.19; $\mathrm{p}=0.026 ; t$ test). Two weeks and two months after the training, the mean step lengths were $0.94 \mathrm{~m}(\mathrm{SD}, 0.21)$ and $0.86 \mathrm{~m}$ (SD, 0.19), respectively, and not significantly different from the step length directly after treatment. Cadence is defined as the number of steps/second. It increased significantly during the training phase (at the end of the baseline phase: mean, $0.80 / \mathrm{s}$; SD, 0.08; at the end of the training phase: mean, $0.87 / \mathrm{s} ; \mathrm{SD}, 0.09 ; \mathrm{p}=0.030$; $t$ test), whereas during the baseline phase no significant

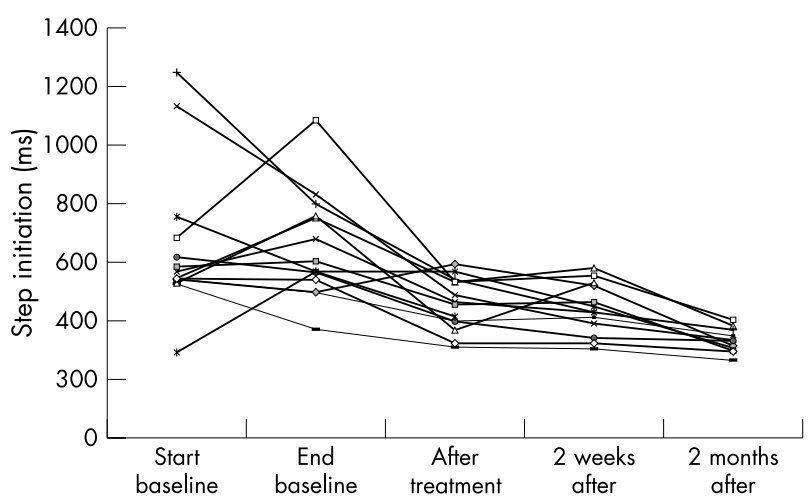

Figure 3 Compensation step initiation in ms; each line represents one patient. Compensation step initiation decreased significantly after treatment. 
changes were seen. At the last follow up, cadence was not significantly different to that seen directly after treatment (mean, 0.86/s; SD, 0.12). Gait velocity showed similar results: no significant changes occurred during the baseline phase, whereas a significant increase was seen during the training phase (at the end of the baseline phase: mean, $0.64 \mathrm{~m} / \mathrm{s}$; SD, 0.20 ; at the end of the training phase: mean, $0.77 \mathrm{~m} / \mathrm{s}$; SD, $0.19 ; \mathrm{p}=0.007 ; t$ test). Two months after training, a mean gait velocity of $0.74 \mathrm{~m} / \mathrm{s}(\mathrm{SD}, 0.19)$ was observed, insignificantly different from the velocity directly after treatment (fig 4). Double support - that is, the time during which both feet contact the floor during walking-is usually prolonged in patients with PD. The repetitive training to make compensatory steps induced a significant reduction of the double support time, which decreased from a mean of $0.19 \mathrm{~s}$ (SD, 0.12 ) at the end of the baseline phase to $0.11 \mathrm{~s}(\mathrm{SD}, 0.16)$ at the end of the training phase $(\mathrm{p}=0.046$; $t$ test $)$. During the follow up period, the time of double support remained nearly constant (two months after training: mean, $0.08 \mathrm{~s}$; SD, 0.10). A limitation of our study is the time resolution of the gait analysis of $20 \mathrm{~Hz}$, which has to be taken into account.

\section{Posturographical testing}

SOT and LOS remained constant during all phases of the study (SOT (table 2); LOS maximal excursion of the centre of gravity in $\%$ of an age matched control group: mean start baseline, $66.1 \%$ (SD, 15.6\%); end baseline 69.7\% (SD, 12.6\%); after treatment $71.3 \%$ (SD, $13.3 \%$ ); after two weeks, $71.7 \%$ (SD, 12.4\%); after two months, $72.5 \%$ (SD, 12.2\%)). Therefore, LOS and SOT were not influenced by the repetitive training of compensatory steps.

\section{PDQ-39}

During all phases of our study, the total PDQ-39 score did not change significantly (start baseline: mean, 28.6 (SD 18.3); end baseline: mean, 25.3 (SD, 15.4); after treatment: mean, 24.5 (SD, 14.8)). However, the subscore mobility decreased significantly after the training (at the end of the baseline phase: mean, $43.6(\mathrm{SD}, 30.1)$; at the end of the training phase: mean, 37.7 (SD, 28.2); $\mathrm{p}=0.015$; Wilcoxon), indicating a subjectively improved mobility. After the follow up period, the mean PDQ-39 score was 25.8 (SD, 20.6) and the mean mobility subscore was 32.1 (SD 25.1) after two weeks and 41.4 (SD, 32.8) after two months, not significantly different from the data directly after treatment.

\section{DISCUSSION}

Because postural instabilities and the associated risk of falling have a strong impact on the prognosis and quality of life of patients with PD, and these impairments are hardly influenced by dopaminergic drugs, a physiotherapeutic

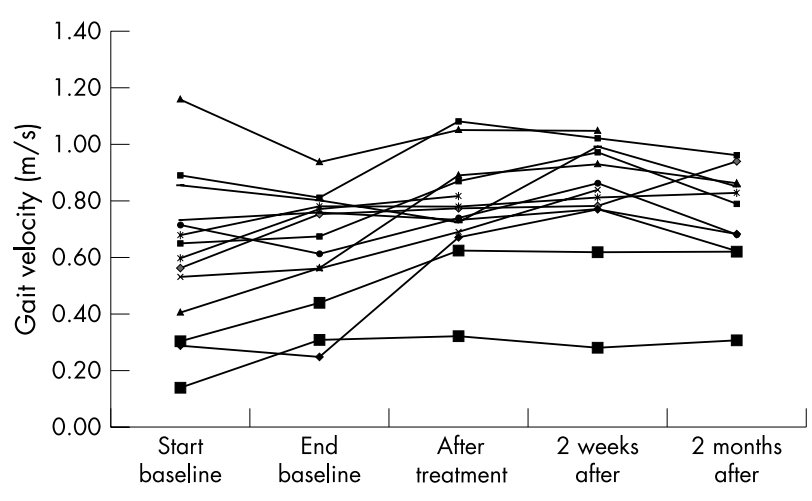

Figure 4 Gait velocity in $\mathrm{m} / \mathrm{s}$; each line represents one patient. Gait velocity significantly increased after treatment. approach is desirable to improve postural reactions and equilibrium.

The results of our present study show that repetitive training of compensatory steps is beneficial for postural and gait parameters in parkinsonian patients: the length of the first compensatory step increased and the time to its initiation shortened. The patients also demonstrated increased walking speed, with a significantly reduced double support time. Finally, the patients' estimation of mobility had improved.

The fact that the compensatory step slightly lengthened during the baseline phase may be explained by the patients' adaptation to the experimental set up (fig 1). Nevertheless, the most prominent and functionally relevant increase of the length of the compensatory step took place during the phase of the repetitive training and the benefit remained stable during the two months of follow up.

Although the patient group was small and heterogeneous - the sexes were mixed, the ages varied widely, and the duration of disease and disease severity were variablethe observed training effects were evident in almost all participants and were very homogeneous, as shown in figs 2 to 4 . Nevertheless, further studies with larger populations are needed to infer from these findings to the general population with PD and postural instability, and include this training as a standard treatment.

Step initiation was measured as the delay between the first movement of the rope very near to the harness and the first movement of the foot. Step initiation consists of two components: (1) approximately a few milliseconds to deliver the impulse via the harness to the body, and (2) "pure" compensatory step initiation time. Step initiation did not change during the baseline phase, whereas it significantly shortened during the training phase. This benefit remained stable during the follow up period. In addition to its helpful influence on postural stability, the repetitive training of compensatory steps influences gait: time of double support decreased, step length and cadence increased. Because both step length and cadence increased, gait velocity naturally increased also. As mentioned above, the time resolution of the gait analysis $(20 \mathrm{~Hz})$ has to be taken into account as a limitation of our study. In particular, mean double support times of $190 \mathrm{~ms}$ at the start of training and $110 \mathrm{~ms}$ at the end of training cannot be distinguished accurately.

Therefore, teaching a patient how to respond to external perturbations while standing leads to an improved gait performance.

Based on posturographical data, Horak proposed that the short stepped, shuffling gait characterising PD may result from a subconscious attempt to keep the centre of the body mass closer to the centre of stability. Following this line of argumentation, the possibility of performing a compensatory step may move this subconscious barrier of the centre of body mass further away from the centre of stability. This mechanism may lead to longer steps and to a shorter double support. ${ }^{14}$

As a consequence of the training, the PDQ-39 mobility subscore increased significantly, reflecting the benefit of the training for the patients' quality of life.

The relatively minor changes in the dependent variables (postural and gait parameters) during the baseline phase, relative to the significant differences exhibited after the training phase, show that the improvements in stability and mobility are attributed to the training rather than pharmacological, psychosocial, and other environmental factors. ${ }^{12}$

To characterise the broad variety of motor strategies to maintain equilibrium, Rogers ${ }^{7}$ proposed that a distinction should be made between corrective and protective responses. During corrective responses, the configuration of the basis of 
Table 2 Sensory organisation test (SOT) data (in degrees/second)

\begin{tabular}{llllll}
\hline Test & Start baseline & End baseline & After treatment & After 2 weeks & After 2 months \\
\hline Eyes open, firm platform & $0.31(0.16)$ & $0.31(0.17)$ & $0.27(0.09)$ & $0.31(0.17)$ & $0.37(0.17)$ \\
Eyes closed, firm platform & $0.54(0.31)$ & $0.50(0.29)$ & $0.46(0.30)$ & $0.55(0.40)$ & $0.62(0.36)$ \\
Eyes open, foam platform & $0.81(0.32)$ & $0.88(0.28)$ & $0.86(0.26)$ & $0.81(0.27)$ & $1.02(0.37)$ \\
Eyes closed, foam platform & $2.24(1.48)$ & $3.28(1.89)$ & $2.31(1.07)$ & $3.33(1.52)$ & $2.69(1.36)$ \\
\hline Values are mean (SD). & & & & \\
\end{tabular}

support-the position of both feet-remains constant, whereas equilibrium is maintained by activation of the leg, trunk, and neck muscles. The impairment of corrective responses is well documented in PD. ${ }^{14-16}$ Dietz et al showed that patients with $\mathrm{PD}$ regulate their stance mainly by modulation of leg flexor activation. In contrast, healthy subjects maintain their equilibrium mainly by activating extensor muscles. The reduced ability to activate leg extensors in patients with PD was proposed to result from an impairment of extensor load receptors. ${ }^{17}$ Therefore, peripheral deficits may play an important part in worsened corrective postural responses in patients with PD. In addition, patients with PD exhibit a reduced excitability within polysynaptic reflexes of the leg extensor muscles, ${ }^{18}$ which may result from deficits at the spinal level. Several aspects of characteristically impaired postural adjustments indicate a defective central programming of the respective electromyograph patterns in PD. This defective central programming may be the result of deficits in the supraspinal control of spinal interneuronal circuits, ${ }^{19}$ and represents impairments at both the spinal and supraspinal level.

In contrast, protective responses are characterised by changes in the base of support (that is, one foot or both feet leave their original position). Protective postural responses are seen when the risk of falling is imminent, independent of a real or a subjectively felt risk. Protective strategies include stepping, grabbing of external supports, and arm reaction responses to absorb the impact of a fall. ${ }^{7}$ It is a common clinical observation that protective responses are diminished or even absent in PD. ${ }^{20} 21$

Compensatory stepping reactions differ in some fundamental ways from volitional stepping. For example, these triggered stepping reactions are initiated and executed much more rapidly than even the fastest efforts to step in response to a visual cue. ${ }^{22}$

Arm reaction studies during compensatory grasping and lower limb reaction studies during compensatory stepping indicate that it is possible for the central nervous system to modulate these triggered reactions to meet environmental demands. ${ }^{23} 24$

The neural substrates that govern the control of rapid perturbation during compensatory stepping also possess a remarkable capacity for immediate and effective recovery of equilibrium. ${ }^{24}$ Clearly, the control is much more complex than the simple "release" of a stereotypical or "ballistic" movement pattern.

In accordance with these observations, Horak et al divide the functional components of postural control into two categories: "motor" and "sensory" components. ${ }^{25}$ Particular sensory components involved in compensatory stepping are plantar cutaneous mechanoreceptors, providing an adequate sensory drive to initiate and control compensatory steps. ${ }^{26}$ In contrast, motor learning is one of the most important "motor" components of postural control. ${ }^{25}$ In accordance with these ideas, we applied recent neurophysiological research findings in stroke patients, suggesting that repetitive motor activity forms an important prerequisite of motor learning, ${ }^{8}$ and transferred these into a new successful therapeutic regimen to treat postural instability in patients with PD.

The influence of dopaminergic medication on central control mechanisms governing compensatory stepping is negligible; therefore, non-dopaminergic lesions are suggested to be involved in the pathophysiology. ${ }^{3}$ Our study population is a proof of this thesis. The patients suffered from postural instability, were aged (mean age, 73 years), and most of them were poorly responsive to L-dopa treatment ( 12 of 14 had less than a $20 \%$ improvement, as indicated by the ratio of values on the UPDRS in the "on" and "off" states; table 1). Such characteristics usually reflect a form of PD with substantial numbers of non-dopaminergic lesions.

Because PD is a widespread degenerative illness, there are several supraspinal candidate regions that are not under dopaminergic influence, may be of high impact for protective responses, and demonstrate longterm plasticity. ${ }^{1-3}$ However, plasticity within the vestibular systems appears to be of minor importance because systematic posturography was not influenced by the training.

In conclusion, postural instability, a common symptom in $\mathrm{PD}$, is only negligibly influenced by antiparkinsonian medication or innovative surgical treatments. If postural instability is evident in the patient's history or clinical examination, repetitive training of compensatory steps can positively influence stability and mobility, thereby improving the patient's quality of life.

\section{Authors' affiliations}

M Jöbges, G Heuschkel, C Pretzel, C Illhardt, C Renner,

H Hummelsheim, Neurologisches Rahabilitationszentrum Leipzig, University of Laipzig, 04828 Bennewitz, Germany

Competing interests: none declared

\section{REFERENCES}

1 Klawans HL, Topel JL. Parkinsonism as a falling sickness. JAMA 1974; 230:1555-7.

2 Koller WC, Glatt S, Vetere-Overfield B, et al. Falls and Parkinson's disease. Clin Neuropharmacol 1989;12:98-105.

3 Bloem BR, Beckley DJ, Dijk van G, et al. Influence of dopaminergic medication on automatic postural responses and balance impairment in Parkinson's disease. Mov Disord 1996;11:509-21.

4 Bronte-Stewart HM, Minn AY, Rodrigues K, et al. Postural instability in idiopathic Parkinson's disease: the role of medication and unilateral pallidotomy. Brain 2002;125:2100-14.

5 Pollak P, Fraix V, Krack P, et al. Treatment results: Parkinson's disease. Mov Disord 2002;(suppl 3):S75-83.

6 Bonnet A-M, Loria Y, Saint-Hilaire $\mathrm{M}-\mathrm{H}$, et al. Does long-term aggravation of Parkinson's disease result from nondopaminergic lesions? Neurology 1987; 37:1539-42.

7 Rogers MW. Disorders of posture, balance, and gait in Parkinson's disease. Clin Geriatr Med 1996;12:825-45.

8 Woldag $\mathrm{H}$, Hummelsheim $\mathrm{H}$. Evidence-based physiotherapeutic concepts for improving arm and hand function in stroke patients. J Neurol 2002;249:518-28.

9 Gibb WRG, Lees AJ. The relevance of the Lewy body to the pathogenesis of idiopathic Parkinson's disease. J Neurol Neurosurg Psychiatry 1988;51:745-52.

10 Fahn S, Elton RL. Members of the UPDRS development committee (1987). Unified Parkinson's Disease Rating Scale. In: Fahn S, Marsden CD, Calne DB, et al, eds. Recent developments in Parkinson's disease, Vol. II. Florham Park: Macmillan Healthcare Information, 1987:153-63, 293-304. 
11 Defer GL, Widner H, Marie RM, et al. Core assessment program for surgical interventional therapies in Parkinson's disease (CAPSI-PD). Mov Disord 1999; 14:572-84.

12 Kazdin AE. Single-case research designs. Methods for clinical and applied settings. New York: Oxford University Press, 1982.

13 Marinus J, Ramaker C, van Hilten JJ, et al. Health related quality of life in Parkinson's disease: a systematic review of disease specific instruments. $J$ Neurol Neurosurg Psychiatry 2002;72:241-8.

14 Horak FB, Nutt JG, Nashner LM. Postural inflexibility in parkinsonian subjects. J Neurol Sci 1992;111:46-58.

15 Stelmach GE, Worringham CJ. Sensorimotor deficits related to postural stability: implications for falling in the elderly. Clin Geriatr Med 1985; 1:679-94.

16 Horak FB, Rank J, Nutt J. Effects of dopamine on postural control in parkinsonian subjects: scaling, set, and tone. J Neurophysiol 1996;75:2380-96.

17 Dietz V, Ziljstra W, Assainte C, et al. Balance control in Parkinson's disease. Gait Posture 1993:1:77-84.

18 Dietz V. Reflex behaviour, a programming in Parkinson's disease. Adv Neurol 1993;60:375-80.
19 Dietz V, Berger W, Horstmann A. Posture in Parkinson's disease: impairment of reflexes and programming. Ann Neurol 1988;24:660-69.

20 Martin JP. The basal ganglia and posture. London: Pitman Publishing, 1976.

21 Bloem BR, Beckley DJ, van Hilten BJ, et al. Clinimetrics of postural instability in Parkinson's disease. J Neurol 1998;245:669-73.

22 Mcllroy WE, Maki BE. Task constraints on foot movement and the incidence of compensatory stepping following perturbation of upright stance. Brain Res 1993;616:30-8.

23 Maki BE, Perry SD, Mcllroy WE. Efficacy of handrails in preventing stairway falls: a new experimental approach. Safety Science 1998;28:189-206.

24 Zettel JL, Mcllroy WE, Maki BE. Can stabilizing features of rapid triggered stepping reactions be modulated to meet environmental constraints? Exp Brain Res 2002; 145:297-308.

25 Horak FB, Shupert CL, Mirka A. Components of postural dyscontrol in the elderly: a review. Neurobiol Aging 1989;10:727-38.

26 Perry SD, Mcllroy WE, Maki BE. The role of plantar cutaneous mechanoreceptors in the control of compensatory stepping reactions evoked by unpredictable multi-directional perturbation. Brain Res 2000:87:401-6.

\section{$\mathrm{ECHO}$}

\section{New management guideline for MHI will be a headache for UK hospitals}

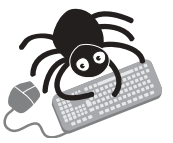

Please visit the Journal of Neurology, Neurosurgery, and Psychiatry website [www.jnnp. com] for a link to the full text of this article.
Wo studies, probably the first in Britain, have suggested that new guidelines for managing minor head injury (MHI) adopted by the National Institute of Clinical

Excellence (NICE) may raise costs, not reduce them as in North America. They predict that there may be major consequences for hospitals without ready access to computed tomography (CT).

Both were studies at Addenbrooke's Hospital, Cambridge, England, a tertiary referral centre for neurosurgery. One was a retrospective case note study of MHI in adults during seven months in the emergency department to compare the number of requests for CT to exclude injury within the skull predicted under the Canadian CT head rule against the actual number and against the number predicted under national guidelines then in force. It concluded that the overall extra cost was 68\%, even allowing for cost savings on skull $x$ ray examinations. Under the Canadian rule nearly $78 \%$ more requests over actual requests would have resulted and $45 \%$ more than under British guidelines, with little clear patient benefit.

The other study was a before and after study of seven months in 2001 and nine months in 2002 to asses the effect of introducing a new protocol based on the Canadian rule, but modified to conserve resources by scrapping night time CT for patients at medium risk in favour of overnight admission for observation and scan if necessary. Essentially, for 12 hours during daytime it conformed to NICE guidelines released in 2003 based on the Canadian rule.

CT scans rose, from $14 \%$ to $20 \%$, a significant but modest rise, and admissions for observation rose too, from $34 \%$ to $45 \%$, though most patients were discharged without needing CT. Skull $x$ ray examinations fell drastically, from $33 \%$ to $1.6 \%$. The ensuing cost increase was not balanced by reduced costs for $x$ ray examinations. Furthermore, the extra expense was conservative, as night time scans had been avoided. This strategy, the study showed, would be a feasible option for hospitals with limited CT resources and is probably low risk, though a larger study would be needed to be sure of safety. Costs were kept within the hospital's resources, but the department intends to continue using its local protocol.

The results were based on 363 useable sets of data in the case note study and 330 and 267 patients with MHI according to the Canadian rule in the before and after stages of the second study, respectively.

MHI accounts for most—up to one million-of those attending hospital with head injuries in the UK a year. The definitive examination for medium or severe head injury is CT, but management of MHI varies enormously around the world.

\ Boyle A, et al. Emergency Medicine Journal 2004;21:426-428.

A Sultan HY, et al. Emergency Medicine Journal 2004;21:420-425. 